\title{
Feasibility of creating a novel animal heart model to test transcatheter techniques for a cavocaval connection that mimics a Fontan completion
}

\author{
Sébastien Gerelli, MD, ${ }^{\mathrm{a}}$ Mathieu Van Steenberghe, MD, ${ }^{\mathrm{a}}$ Mehul Patel, MD, ${ }^{\mathrm{a}}$ Isabelle Van Aerschot, MD, ${ }^{\mathrm{a}}$ and \\ Younes Boudjemline, $\mathrm{MD}, \mathrm{PhD}^{\mathrm{a}, \mathrm{b}}$
}

\begin{abstract}
Objective: The objective of this study was to create a novel animal model to foster the future development of interventional techniques for a cavocaval connection that mimics a Fontan completion.

Methods: Ten sheep were studied. All had the superior vena cava-right atrium junction closed using a polytetrafluoroethylene membrane. A valveless Gore-Tex conduit connecting the terminal portion of the superior vena cava to the right atrium was used to bypass the polytetrafluoroethylene occlusion and to allow normal venous drainage through the right atrium. Radio-opaque nitinol rings were placed around the inferior vena cava near its entry in the right atrium to allow better fluoroscopic visualization and to enhance stent stability during transcatheter cavocaval connection.
\end{abstract}

Results: The first 3 animals died during the learning curve as a result of technical issues. The subsequent 7 surviving sheep showed good flow dynamics on cardiac catheterization. Transcatheter cavocaval connection was performed successfully performed in 6 sheep at 0 to 9 months after the initial surgery. Sacrifice was done electively in all animals at 1 to 9 months per protocol. One animal was euthanized early because of an untreatable infection. One sheep was observed as a control without a transcatheter cavocaval connection and was sacrificed at 12 months.

Conclusions: A novel, chronic ovine model to foster development of transcatheter techniques for cavocaval connection to mimic a Fontan completion was created. The successful model is easily reproducible after a short learning curve and shows good survival. (J Thorac Cardiovasc Surg 2013;146:408-12)

Children with functional univentricular hearts face remarkable challenges during their lifetime. The development of the Fontan operation has improved survival significantly beyond the fourth decade; however, multiple, staged surgeries affect postoperative quality of life negatively in such patients. The complexity and the broad scope of improvement of the univentricular heart have inspired some highly creative surgical techniques to prepare the heart for subsequent transcatheter repair. ${ }^{1-10}$ The interest to perform transcatheter completion of Fontan stems from the need to reduce the number of surgeries in these patients. The interventional armamentarium with the advent of stents has revolutionized the field of pediatric cardiology drastically. The use of stents

\footnotetext{
From the Centre de Référence Malformations Cardiaques Congénitales ComplexesM3C ${ }^{\text {a }}$ Necker Hospital for Sick Children, Assistance Publique des Hopitaux de Paris, Pediatric Cardiology, Paris, France; and the Université Paris Descartes, ${ }^{\mathrm{b}}$ Sorbonne Paris Cité, Paris, France.

This work was supported by the Fondation de l'Avenir.

Disclosures: Authors have nothing to disclose with regard to commercial support.

Received for publication March 23, 2012; revisions received Sept 10, 2012; accepted for publication Sept 21, 2012; available ahead of print Oct 24, 2012.

Address for reprints: Younes Boudjemline, MD, PhD, Necker Hospital For Sick Children, Pediatric Cardiology, 149 rue de Sèvres, 75015 Paris Cedex, France (E-mail: younes.boudjemline@nck.aphp.fr).

$0022-5223 / \$ 36.00$

Copyright (c) 2013 by The American Association for Thoracic Surgery

http://dx.doi.org/10.1016/j.jtcvs.2012.09.059
}

has evolved from simple a bailout of a stenosis or obstruction to an alteration of flow dynamics to achieve optimal physiology. Despite several experimental studies published on the development of surgical preparation and transcatheter Fontan completion, there is conspicuous lack of data on the creation of a viable, chronic animal model for testing transcatheter techniques for cavocaval connection in Fontan completion. The nonviable models reported in the past failed to show long-term assessment of the surgical pathway. ${ }^{7,8}$ Delayed and long-term complications such as thrombus formation, device fracture, failure, and so forth, remain unstudied.

We report the feasibility for creating a chronic, viable ovine heart model. Our study was intended to simulate a surgical substrate of partial cavopulmonary circulation and to describe the challenges faced during the evolution and creation of the surgical model that may allow development and long-term assessments of newer techniques for transcatheter cavocaval connection.

\section{METHODS}

Our study was performed in accordance with the Guidelines for the Care and Use of Laboratory Animals and was approved by the Subcommittee on Research Animal Care and the local institutional ethics committee (INRA, Paris, France). ${ }^{11}$ All animals received humane care in compliance with the European Convention on Animal Care. 


\section{Abbreviations and Acronyms \\ IVC = inferior vena cava \\ PA = pulmonary artery \\ PTFE $=$ polytetrafluoroethylene \\ $\mathrm{RA}=$ right atrium \\ $\mathrm{SVC}=$ superior vena cava}

\section{Surgical Technique}

Ten consecutive sheep were studied after improvisation of surgical technique from an earlier experience. Standard premedication, anesthesia induction, endotracheal intubation, and ventilation were achieved in all sheep. Anesthesia was maintained with $2 \%$ halothane. The heart was exposed through a conventional right thoracotomy and no extracorporeal circulation was used. All sheep underwent total interruption of the superior vena cava (SVC)-right atrium (RA) junction by a polytetrafluoroethylene (PTFE) membrane. A nitinol wire was preshaped to an open ring and sized with approximation to fit the inferior vena cava (IVC) snuggly. Two open nitinol rings were placed around the IVC distal to the opening of the hepatic veins and near its entry to the RA. The rings served as radio-opaque markers for precise placement as well as an anchor of a covered stent for subsequent transcatheter completion. All sheep underwent placement of a valveless Gore-Tex conduit that connected the terminal portion of the SVC to the RA to divert and bypass the PTFE occlusion and allow normal venous drainage.(Figure $1, D$ ).

\section{Transcatheter Completion}

We planned to perform transcatheter completion at 1, 3, 6, and 9 months after the initial surgery. One animal was planned as a control with no transcatheter completion. The technique of completion has been reported previously. ${ }^{8}$ Briefly, cardiac catheterization was performed using both the right femoral and internal jugular veins. A catheter was placed in contact with the membrane separating the SVC and the RA. The stiff end of a guidewire was pushed through the membrane. After perforation of the membrane, a balloon catheter was inflated at the level of the membrane. Several covered stent grafts mounted on a 22-mm balloon catheter were deployed to bridge the gap between the opened SVC and the radio-opaque nitinol rings at the IVC-RA junction. Selective angiographies were performed to confirm the absence of flow from the SVC to the RA, after reestablishing the pathway between the SVC and the RA, and after transcatheter cavocaval connection.

\section{Sacrifice}

Per study protocol, euthanasia was planned at 1,3,6, and 9 months after completion, or at 12 months after surgical preparation in the animal without transcatheter completion.

\section{RESULTS}

Ten sheep were used in the study. The first 3 died early as a result of technical issues. Two died from hypovolemic shock secondary to uncontrolled hemorrhage (accidental rupture of anastomosis) before transcatheter completion and 1 died from SVC syndrome as a result of occlusion of the SVC-RA connection after transcatheter completion. The remaining 7 sheep survived the operative procedure and recovered uneventfully. All animals except one underwent transcatheter cavocaval completion at 0 to 9 months after surgery. All animals except one survived transcatheter completion and were followed 1 to 9 months after the completion (median, 5 months). One animal experienced fever, weight loss and poor feeding after completion secondary to a fulminating infection. The animal was euthanized after 1 week of ineffective treatment with antibiotics. The uncompleted animal was left alive and euthanized 1 year after the initial surgery. Early and late catheterization was done in all surviving sheep and all demonstrated good flow dynamics, with a mean gradient across the SVC pathway between $0 \mathrm{~mm} \mathrm{Hg}$ and $2 \mathrm{~mm} \mathrm{Hg}$.

Cardiac catheterization during the completion steps with assessment of patency of the SVC-RA connection, PTFE membrane perforation, and thus reestablishment of the native SVC-RA connection is highlighted in Figure 2. Subsequent angiographic views after completion showing the covered stents connecting the SVC and the IVC, with redirection of IVC flow, are presented in Figure 3.

All sheep (except the one described previously) euthanized per protocol demonstrated good health at the time of sacrifice. At macroscopic examination, no thrombus was found at the SVC-RA junction, which remained widely open. In completed animals, all stents were patent with no leakage or thrombus formation (Figure 4). No thrombus was noted in the lungs.

\section{DISCUSSION}

Hausdorf and colleagues ${ }^{1,2}$ were the first to attempt and describe transcatheter completion of a hemi-Fontan using balloon dilatation of the banded cavoatrial junction and fenestration closure using either a covered stent or patent ductus arteriosus occluders. Subsequently, Klima and colleauges ${ }^{3}$ developed a new approach by establishing bidirectional Glenn circulation and subtotal banding of the SVC at the cavoatrial junction, along with PTFE cuff placement around the IVC - all performed in the absence of cardiopulmonary bypass. To facilitate placement of the graft stent in the SVC-RA junction, and to avoid residual obstruction around the SVC band site, Galantowicz and Cheatham ${ }^{4,5}$ closed the SVC-RA junction by using a pericardial patch for a subsequent intracardiac stent. Konstantinov and others ${ }^{6,7}$ modified this further by placing stents at the level of both atriocaval junctions. The atrial SVC was then connected to the inferior face of the unopened right pulmonary artery (PA). The stent at the SVC-PA junction may cover a part of the PA lumen and thus reduce the forward flow. With kinetic energy as the only force to drive the blood in a circuit with resistance in series, and absent a driving pump, any degree of loss of energy reduces cardiac output and vital organ perfusion.

Despite such promising preliminary results of transcatheter completion of Fontan, there are some unanswered questions about the ideal surgical technique to prepare a univentricular heart for subsequent transcatheter techniques. The insertion of foreign material inside the heart, 


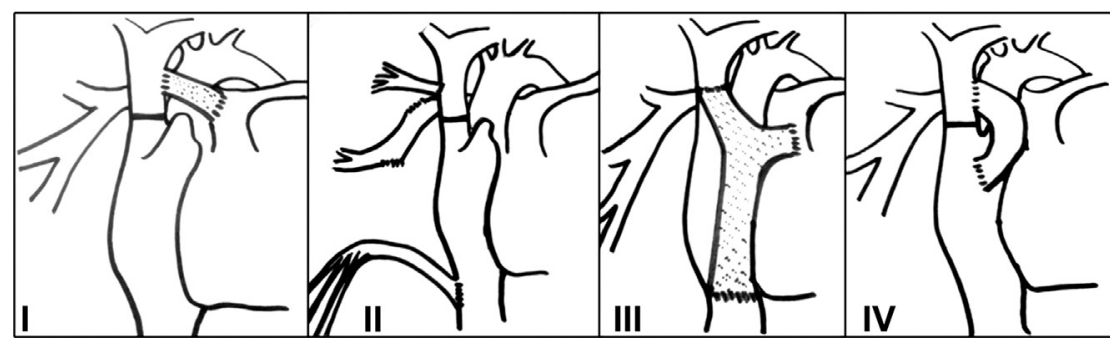

FIGURE 1. Schematic drawings showing all techniques used for the creation of a simulated cavopulmonary sheep heart model. This study presents only the results from view D. Techniques depicted in views A through C resulted in $100 \%$ mortality.

along with the iatrogenic dead space, may favor thrombus formation. Given the fact that univentricular hearts have open connection between the right and left circulation, any thrombus formation in any chamber can be catastrophic. There is a growing need to have a chronic animal heart model to assess such potential complications and the long-term outcomes of transcatheter techniques. In our prior experience we tested various surgical models to simulate Fontan physiology (data not shown). The surgical techniques used included placement of a valveless conduit between the SVC and the main PA; side-to-side bicaval branch PA anastomosis, with the right upper PA branch anastomosed to the SVC and the right lower lobar branch anastomosed to the IVC; and total cavopulmonary bypass with a valveless synthetic $\mathrm{Y}$ tube between the 2 vena cava and the main pulmonary artery.

Creation of such surgical models with physiologic simulation of cavopulmonary circulation was highly eventful with no survival beyond 2 hours of surgery (Figure 1, A, $B$, and $C$ ). It was realized that animals would not tolerate bypassing the right ventricle even for a couple hours. To circumvent the problems noted with our early experience, we modified our surgical approach significantly and developed an anatomic model as a platform to test the technical feasibility and the long-term outcomes of transcatheter cavocaval connection for Fontan completion (Figure 1,D).
The model from the current study was therefore designed to redirect flow without altering the cardiac architecture or physiology. The results were spectacular and allowed us to test the completion of the cavocaval connection chronically in all animals per protocol.

\section{Clinical Implications}

We believe our surviving animal anatomic model closely mimics the pre-Fontan preparatory stage of a univentricular heart from an interventional standpoint. The uniform survival with only planned deaths after a short learning curve makes this a good and a reproducible technique. This working model should be suitable to test various transcatheter therapies, including the possibility of hybrid therapies in the future. ${ }^{9,10}$ With greater experience it may be possible to create a hybrid strategy with the current model to accomplish intraoperative completion of Fontan in the same setting with significant reduction of crossclamp time and perioperative mortality. We believe this is the only pathway that works well and has good long-term survival. All attempts to create a real-life anatomic and physiologic cavopulmonary connection failed with $100 \%$ mortality. The current model proved to be ideal in testing various transcatheter therapies for completion. Each step of the surgical preparation and the subsequent transcatheter completion can be tested with reliable accuracy, including surgical

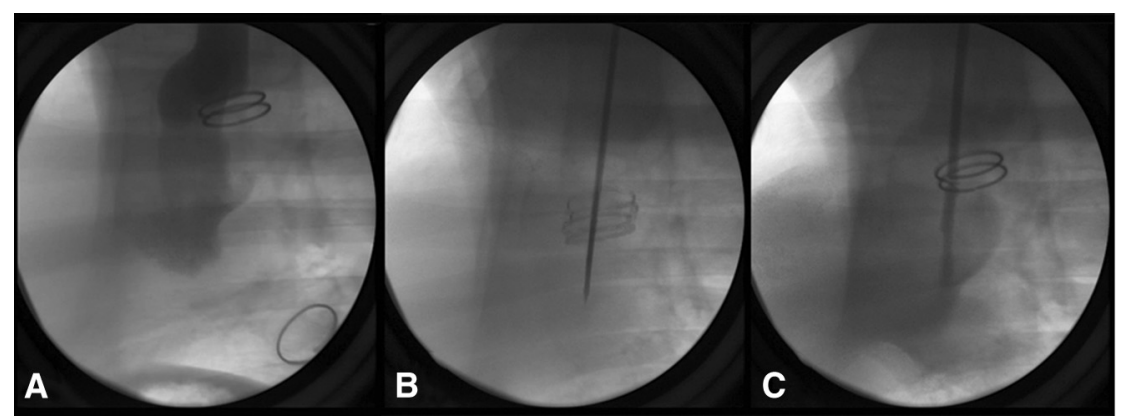

FIGURE 2. Angiographic views showing the perforation steps of the Fontan completion. A, Preliminary dye injection shows the surgical pathway in the animal. The superior vena cava has been connected to the right atrium. The normal superior vena cava-right atrium connection is occluded by a polytetrafluoroethylene membrane. Two rings have been placed around the superior vena cava at the level of the membrane and 2 around the inferior vena cava (only 1 is shown here). B, Perforation of the membrane with a needle. C, Dye injection in the sheath after perforation shows the reestablished connection between the superior vena cava and the right atrium. 

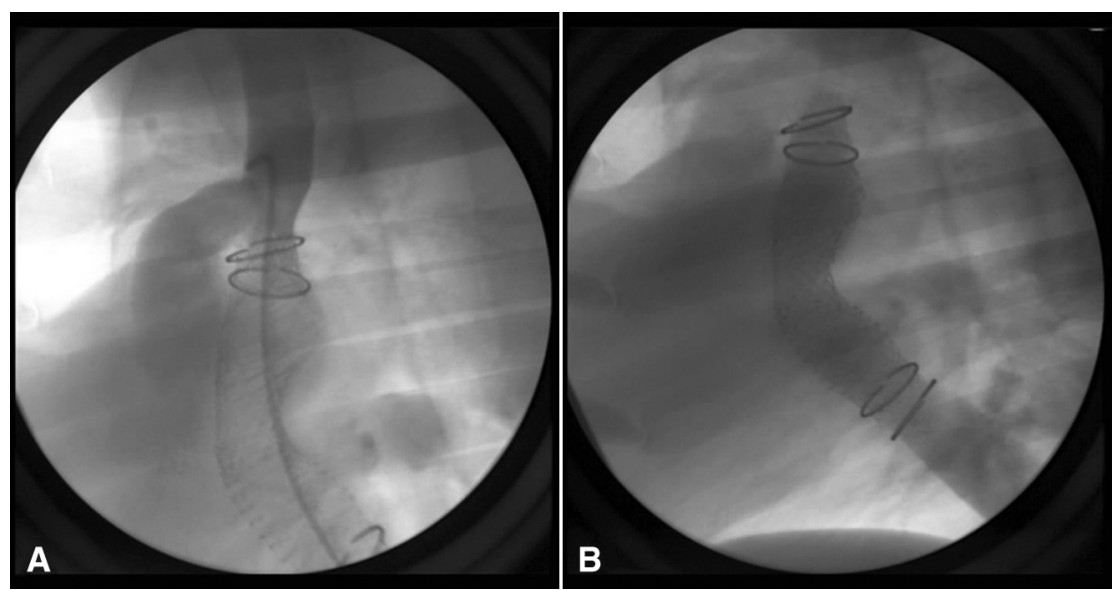

FIGURE 3. Angiographic views after completion. A, Covered stents have been placed between the superior vena cava and the inferior vena cava. Dye injection in the superior vena cava shows the connection to the right atrium. B, Dye injection in the inferior vena cava shows the contrast going into the covered stents in the superior vena cava connection.

creation of superior connection, placement of markers around the IVC, pliability of the SVC-RA PTFE membrane for various perforation techniques and transcatheter opening of the occluded SVC, and covered stent placement. Moreover, because it is a viable model without significant physiologic alterations, long-term complications can also be assessed and may include thrombus formation secondary to a foreign body and dead space; complications of stent fracture, migration, and dislodgment; damage to the IVC and neighboring structures; impact of the tendinous insertion of the diaphragm at the caval opening with its dynamic motion on the stent; and paraprosthetic leak.

\section{Study Limitations}

The model we created is not a physiologic model of Fontan circulation because all animals had structurally normal hearts with a well-developed right ventricle pumping the right circulation. However, it is a good simulated platform to test various transcatheter and hybrid techniques. The potential of thrombus formation in the long run still cannot be ruled out completely; however, none of the surviving sheep had any thrombus formation as of the last follow-up. Moreover, for future clinical applications, a definitive anticoagulation strategy may be required that was not used in any of our animals.

\section{CONCLUSIONS}

We describe new surgical technique for the preparation for an anatomic ovine heart model for transcatheter completion of Fontan circulation. This surgical technique was easily reproducible after a short learning curve with no major limitations. The novel and successful creation of a viable

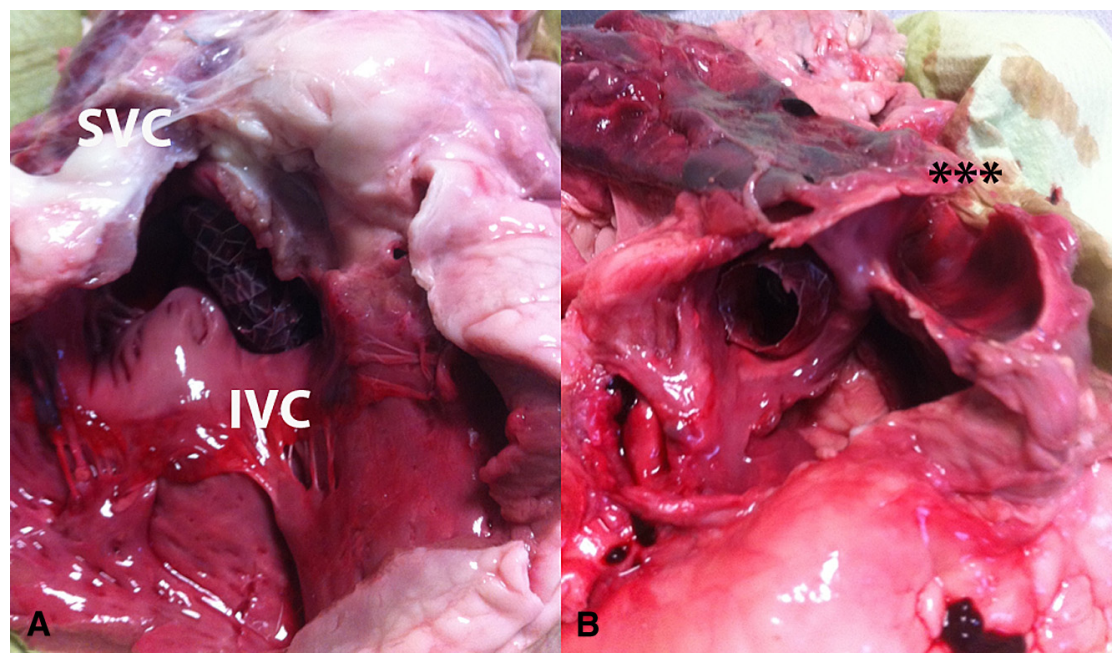

FIGURE 4. Gross examination of the heart after sacrificing the animal. A, The covered stent running from the superior vena cava (SVC) to the inferior vena cava $(I V C)$. B, The connection between the SVC and the right atrium after retrieval of the IVC stent $(* * *)$. 
ovine heart model marks a new milestone in allowing further evaluation of transcatheter and hybrid techniques in univentricular hearts. Additional long-term studies with this model may be required before it may be implemented clinically.

We thank the Fondation de l'Avenir for its financial support.

\section{References}

1. Hausdorf G, Schneider M, Konertz W. Surgical preconditioning and completion of total cavopulmonary connection by interventional cardiac catheterization: a new concept. Heart. 1996;75:403-9.

2. Konertz W, Schneider M, Herwig V, Kampmann C, Waldenberger F, Hausdorf G Modified hemi-Fontan operation and subsequent nonsurgical Fontan completion. J Thorac Cardiovasc Surg. 1995;110:865-7.

3. Klima U, Peters T, Peuster M, Hausdorf G, Haverich A. A novel technique for establishing total cavopulmonary connection: from surgical preconditioning to interventional completion. J Thorac Cardiovasc Surg. 2000;120: 1007-9.
4. Cheatham JP, Galantowicz M, Torres W, Tower AJ, Hill SL, Kleinman CS, et al. The use of custom-made covered NuMed CP stents in the treatment of congenital heart disease. Catheter Cardiovasc Interv. 2002;57:100-1.

5. Galantowicz M, Cheatham JP. Fontan completion without surgery. Semin Thorac Cardiovasc Surg Pediatr Card Surg Annu. 2004;7:48-55.

6. Konstantinov IE, Meskishvili VV. Intracardiac covered stent for transcatheter completion of the total cavopulmonary connection: anatomical, physiological and technical considerations. Scand Cardiovasc J. 2006;40:71-5.

7. Konstantinov IE, Benson LN, Caldarone CA, Li J, Shimizu M, Coles JG, et al. A simple surgical technique for interventional transcatheter completion of the total cavopulmonary connection. J Thorac Cardiovasc Surg. 2005;129:210-2.

8. Metton O, Calvaruso D, Stos B, Ben Ali W, Boudjemline Y. A new surgical technique for transcatheter Fontan completion. Eur J Cardiothorac Surg. 2011;39:81-6.

9. Hijazi ZM. Intraoperative intervention (hybrid surgery) and intervention in the immediate perioperative period. Catheter Cardiovasc Interv. 2003;60:99-100.

10. Bacha EA, Marshall AC, McElhinney DB, del Nido PJ. Expanding the hybrid concept in congenital heart surgery. Semin Thorac Cardiovasc Surg Pediatr Card Surg Annu. 2007;146-50.

11. Council of Europe. European convention for the protection of vertebrate animals used for experimental and other scientific purposes. Council of Europe Convention ETS No. 123 and Directive 86/609/EEC. Strasburg, Germany; March 18, 1986. 